Review Article

\title{
Primary Nephrotic Syndrome in Adults as a Risk Factor for Pulmonary Embolism: An Up-to-Date Review of the Literature
}

\author{
Aibek E. Mirrakhimov, ${ }^{1}$ Alaa M. Ali, ${ }^{1}$ Aram Barbaryan, \\ Suartcha Prueksaritanond, ${ }^{1}$ and Nasir Hussain ${ }^{1,2}$ \\ ${ }^{1}$ Department of Internal Medicine, Saint Joseph Hospital, 2900 N. Lake Shore, Chicago, IL 60657, USA \\ ${ }^{2}$ College of Physicians and Surgeons, Columbia University, New York-Presbyterian Hospital, New York, NY 10034, USA \\ Correspondence should be addressed to Aibek E. Mirrakhimov; amirrakhimov1@gmail.com
}

Received 12 February 2014; Accepted 7 April 2014; Published 16 April 2014

Academic Editor: Jochen Reiser

Copyright (C) 2014 Aibek E. Mirrakhimov et al. This is an open access article distributed under the Creative Commons Attribution License, which permits unrestricted use, distribution, and reproduction in any medium, provided the original work is properly cited.

Patients with nephrotic syndrome are at an increased risk for thrombotic events; deep venous thrombosis, renal vein thrombosis, and pulmonary embolism are quite common in patients with nephrotic syndrome. It is important to note that nephrotic syndrome secondary to membranous nephropathy may impose a greater thrombotic risk for unclear reasons. Increased platelet activation, enhanced red blood cell aggregation, and an imbalance between procoagulant and anticoagulant factors are thought to underlie the excessive thrombotic risk in patients with nephrotic syndrome. The current scientific literature suggests that patients with low serum albumin levels and membranous nephropathy may benefit from primary prophylactic anticoagulation. A thorough approach which includes accounting for all additional thrombotic risk factors is, therefore, essential. Patient counseling regarding the pros and cons of anticoagulation is of paramount importance. Future prospective randomized studies should address the question regarding the utility of primary thromboprophylaxis in patients with nephrotic syndrome.

\section{Introduction}

Nephrotic syndrome (NS) is characterized by proteinuria of $\geq 3.5 \mathrm{~g} / 24$ hours, albuminemia $<3.0 \mathrm{~g}$, peripheral edema, hyperlipidemia, lipiduria, and increased thrombotic risk [ 1 , 2]. The etiology of NS is divided into primary NS and secondary NS. Furthermore, secondary causes of NS can be subdivided into NS-related systemic diseases and NS related to medication use. Common primary causes of NS are focal segmental glomerulosclerosis (FSGS), membranous nephropathy $(\mathrm{MN})$, and minimal change disease (MCD) (after excluding identifiable causes such as cancer, systemic diseases, and medications) [2]. Common causes of NS secondary to systemic diseases are diabetes mellitus, systemic lupus erythematosus, multiple myeloma, amyloidosis, malignancy, and infections [2]. Pamidronate, nonsteroidal antiinflammatory drugs, penicillamine, and gold compounds are among the most commonly implicated medications in the development of NS [2].
Primary NS is a relatively rare entity compared to NS secondary to systemic diseases, particularly diabetes mellitus [2]. FSGS and MN are the more common pathological forms of NS in adults, whereas MCD is more common in the pediatric population. Some cases of immunoglobulin A nephropathy (IgAN) and membranoproliferative glomerulonephritis may clinically present with NS [2-4]. It is important to note that renal biopsy is essential in making a diagnosis and in certain diseases (such as systemic lupus erythematosus) it guides the therapy [5].

Pulmonary embolism (PE) is a serious complication of deep venous thrombosis (DVT), with a significant morbidity and mortality $[6,7]$. Common risk factors for the development of DVT and PE are prolonged immobilization, recent surgery, cancer, cardiac disease, autoimmune disease (such as inflammatory bowel disease), and prior history of DVT/PE and hypercoagulable conditions (such as NS and antiphospholipid antibody syndrome) $[8,9]$. 
The clinical presentation of PE can range from asymptomatic to tachypnea and tachycardia to cardiovascular collapse and death [6]. Moreover, episodes of PE can have longterm consequences such as chronic thromboembolic pulmonary hypertension (CTEPH) [10]. High clinical suspicion is, therefore, paramount in the diagnosis of $\mathrm{PE}$.

The goal of this paper is to summarize the scientific data regarding the impact of primary NS on the risk of PE. We will review the current understanding of the pathophysiology of increased thrombotic risk among patients with NS. Second, clinical data and thrombosis predictors will be reviewed. Third, we will review the current data on the utility of prophylactic anticoagulation. However, we will not provide a detailed review on the clinical presentation, imaging, and treatment of PE, since these topics are out of the paper's scope and can be easily found elsewhere [6].

\section{Why Do Patients with Nephrotic Syndrome Are at Increased Risk for Thrombotic Events?}

NS on a tissue level represents damage to the glomerulus, with resultant dysfunction and permeability to various endogenous substances which are not filtered through the glomerular membrane under normal physiologic conditions [2]. Most features of NS are directly related to the increased glomerular permeability, for example, proteinuria, hypoalbuminemia, and lipiduria. The hypercoagulable state seen in patients with primary NS is believed to be secondary to glomerular pathology. However, patients with malignancyrelated NS are likely to represent a group who is at even greater risk for thrombotic events due to underlying cancer [11]. Below, we will review the current concepts of increased thrombotic risk among patients with primary NS.

Patients with NS have increased platelet reactivity and often thrombocytosis [12-15]. The pathophysiology of platelet hyperactivity is not entirely understood, but several key factors are believed to contribute. First, it is well known that thromboxane A2 (TxA2) is a major promoter of platelet activation and clot formation [16]. Arachidonic acid (AA), which is a precursor for TxA2 synthesis, is released from cells in a constant fashion [17]. Albumin binds AA, thus, making it unavailable for platelet metabolism and conversion into TxA2; therefore, TxA2 levels are increased in patients with NS because of hypoalbuminemia, thereby favoring clot formation and platelet hyperactivity [18-21]. Second, elevated fibrinogen levels seen in patients with NS can promote platelet aggregation [22]. Third, elevated cholesterol (commonly seen in patients with NS) can promote platelet aggregation $[23,24]$. Finally, patients with NS have increased levels of Von Willebrand factor (vWf) and decreased red blood cell membrane flexibility, which promote platelet adhesion [22].

It is interesting to note that the aggregation of red blood cells may be increased in patients with NS and, thus, may contribute to thrombogenesis $[25,26]$. This phenomenon is believed to be secondary to hypoalbuminemia, intravascular volume depletion, red blood cell dehydration (secondary to hypernatremia), and increased fibrinogen levels.

At the level of the coagulation system, several events lead to the thrombotic risk seen in patients with NS. First, antithrombin III (ATIII) levels are decreased in patients with NS. ATIII is a potent endogenous antithrombotic substance and a major factor responsible for the clinical activity of heparin, which targets several coagulation factors such as factor II, factor VII, factor IX, factor X, and factor XII [27]. Urinary loss of ATIII secondary to glomerular membrane permeability is believed to be a principal source of decreased ATIII levels in patients with NS [28-30]. Another mechanism for decreased levels of ATIII observed in patients with NS can be constantly ongoing subclinical thrombosis with the consumption of ATIII [31]. Second, protein S activity may be impaired in patients with NS. Protein $S$ is an essential vitamin $\mathrm{K}$ dependent cofactor of protein $\mathrm{C}$ that is involved in the inactivation of coagulation factor $\mathrm{V}$ and factor VII [32]. It is interesting to note that protein $S$ levels in patients with NS may be increased [33]. However, protein $S$ is present in two forms, free (active) and protein-bound (inactive), with the active form being lost in the urine of patients with NS compared to the inactive form $[34,35]$. Therefore, most of the measured protein S in patients with NS is the protein-bound form, which is not physiologically active. However, certain anticoagulation factors such as protein $\mathrm{C}$ and tissue factor pathway inhibitor are preserved in patients with NS, which is believed to compensate for the procoagulant state seen in NS $[36,37]$. However, these results were observed in pediatric patients and it is not clear whether they can be extrapolated to adult patients with NS. Nevertheless, the above-mentioned compensatory increase in protein $\mathrm{C}$ and tissue factor pathway inhibitor is inadequate to counteract the increased risk of thrombosis in patients with NS.

On the other hand, fibrinogen levels are increased in patients with NS [38]. This increase is believed to be mediated by hypoalbuminemia which in turn increases hepatic fibrinogen synthesis. As mentioned above, fibrinogen enhances platelet reactivity and red blood cell aggregation [22, 39]. Furthermore, patients with NS have greater levels of coagulation factor V, factor VII, and alpha-2 macroglobulin, which is believed to be secondary to upregulated production [39]. However, levels of coagulation factor XI were reported to be decreased in children with NS [40]. Decreased levels of factor XI may be protective against thrombotic events since elevated level of factor XI portends a greater risk of thrombosis in patients without NS [41].

Patients with NS have reduced fibrinolytic activity [42] and increased urinary losses of plasmin, which is a key fibrinolytic protein [43]. In addition, increased levels of lipoprotein a (Lpa) in patients with NS may further counteract fibrinolytic activity [44]. Moreover, patients with NS have increased levels of plasminogen activator inhibitor-1, which is a natural inhibitor of the conversion of plasminogen to plasmin [45]. It is essential to note that fibrin clots in patients with NS may be more resistant to fibrinolysis because of lower thrombus porosity [46]. Furthermore, from a theoretical point of view, it is possible that diuretic use (used to mitigate NS associated body edema) in patients with 
NS may potentiate hemoconcentration, which, in turn, will promote clot formation.

Future studies should explore the mechanisms responsible for the increased thrombotic risk seen in NS secondary to MN. A simplified overview of the pathogenesis of NS-related thrombotic risk is presented in Figure 1.

\section{Clinical Predictors of Thrombotic Risk in Patients with NS}

It is essential to keep in mind that many patients with NS may have other risk factors for venous thrombosis besides NS. Such risk factors include prolonged immobilization, recent surgery, prior DVT and PE, obesity, the presence of central venous catheters, stroke, and palsies [8]. Thus, it is essential to approach the evaluation of thrombotic risk in these patients in a thorough manner.

Several clinically useful predictors are of utility in stratifying patients with NS regarding the future risk of thrombotic events. First, histologic diagnosis of NS is of paramount importance in assessing the risk of thrombosis. Barbour et al. analyzed the data of 1,313 patients with idiopathic NS (395 subjects with MN, 370 subjects with FSGS, and 548 subjects with IgAN) [47] and demonstrated that the diagnosis of $\mathrm{MN}$ was associated with an increased risk of venous thromboembolism (VTE) compared to FSGS and IgAN (more than 2-fold increased risk compared to FSGS and more than 19-fold increased risk compared to IgAN). Lionaki et al. studied 898 patients with biopsy proven $\mathrm{MN}$ to assess possible predictors for increased thrombotic risk [48]. These investigators showed that an albumin level $<2.8 \mathrm{~g} / \mathrm{L}$ was independently associated with a higher thrombotic risk. Moreover, every $1.0 \mathrm{~g} / \mathrm{L}$ reduction in albumin was translated into a 2.13-fold increased risk of VTE.

Causes of NS other than MN may impose a lesser risk for thrombosis, and, in such groups, a lower level of albumin may be associated with thrombotic risk. Cherng et al. demonstrated that patients with NS and an albumin level $<2.0 \mathrm{~g} / \mathrm{L}$ had a greater risk of VTE and PE [49]. It is essential to point out that no data on histological diagnosis were available in their study. Another interesting finding of their study was that $29 \%$ of patients had evidence of PE, with some of these cases being asymptomatic. Kuhlmann et al. also showed that an albumin level $<2.0 \mathrm{~g} / \mathrm{L}$ was associated with an increased thrombotic risk among patients with NS [50].

Age is an important risk factor for VTE among patients with NS since adult patients have an approximately 7- to 8 -fold increased risk of a thrombotic event compared to children with NS $[2,51]$.

Therefore, based on the above data, it is essential to have a thorough approach for the analysis of thrombotic risk in patients with NS. Consideration of conventional risk factors for DVT and VTE is of paramount importance. Several specific clinical markers are of clinical use, such as a biopsy proven diagnosis of $\mathrm{MN}$, albumin level $<2.8 \mathrm{~g} / \mathrm{L}$ in patients with $\mathrm{MN}$, and albumin level $<2.0 \mathrm{~g} / \mathrm{L}$ in NS other than $\mathrm{MN}$.

\section{Clinical Presentation and Epidemiology of PE in Patients with NS}

DVT is a major risk factor for PE [8]; lower extremities are the major site for DVT occurrence [52]. The most common symptoms of DVT are extremity swelling, erythema, and pain. Given its associated morbidity and mortality, it is essential to consider DVT in the differential diagnosis in patients who present with new onset extremity pain (especially when nontrauma related), swelling, and redness. Several clinical score systems are available to help clinicians stratify the risk of a possible DVT; Wells score for DVT is among the most commonly used ones [53] and is presented in Table 1. In patients with a low probability of DVT, a negative D-dimer test rules out DVT in most, but not in all patients [54]. In patients with a Wells score $\geq 1$, Doppler ultrasound of lower extremities must be performed to exclude DVT [53]. In all patients with confirmed DVT, anticoagulation therapy is warranted and if medically contraindicated, an inferior vena cava (IVC) filter should be placed [52].

As mentioned above, DVT is a major risk factor for PE [8]. The clinical presentation of PE ranges from asymptomatic to nonspecific complaints of shortness of breath and chest pain to cardiovascular collapse and death [6]. A modified version of Wells score for $\mathrm{PE}$ is available for clinical use to help clinicians stratify the probability of PE [55]. Wells score for PE is presented in Table 2. A negative D-dimer in patients with a Wells score for $\mathrm{PE} \leq 4$ (some argue for $\leq 2$ ) effectively excludes $\mathrm{PE}$ in most patients. Patients with a greater score or positive D-dimer should undergo computed tomography (CT) of the chest with the administration of intravenous contrast [8]. However, poor renal function (defined as elevated creatinine and/or decreased glomerular filtration rate), contrast allergy, and the simple lack of a CT machine may preclude this useful imaging modality in some patients. In such circumstances, ventilation-perfusion nuclear scan (V/Q) may be useful; a negative V/Q scan rules out PE and low probability of V/Q scan with a low clinical probability of PE is useful in excluding the disease; all other combinations generally require further testing. However, it is important to remember that V/Q scans have decreased sensitivity and specificity in patients with underlying pulmonary disease [10].

The management of $\mathrm{PE}$ is complex and depends on various factors, such as cardiovascular instability and the presence of right ventricular dysfunction. The reader is referred to focused reviews on this topic $[8,56]$.

DVT is the most common thrombotic complication of NS according to some studies. Kayali et al. studied 925,000 patients with NS and compared them to 898,253 patients without NS [57]. These researchers found that patients with NS had a greater risk for both DVT and PE, with a relative risk of 1.72 and 1.39 , respectively. In contrast to them, Suri et al. showed that PE was more common than DVT (25.7 versus $16.6 \%$, resp.) [58]. However, the study sample included only 34 pediatric patients with NS. This actually may explain the different findings in regard to the commonest thrombotic complication of NS.

It is important to keep in mind, however, that patients with NS also have an increased risk of renal vein thrombosis 


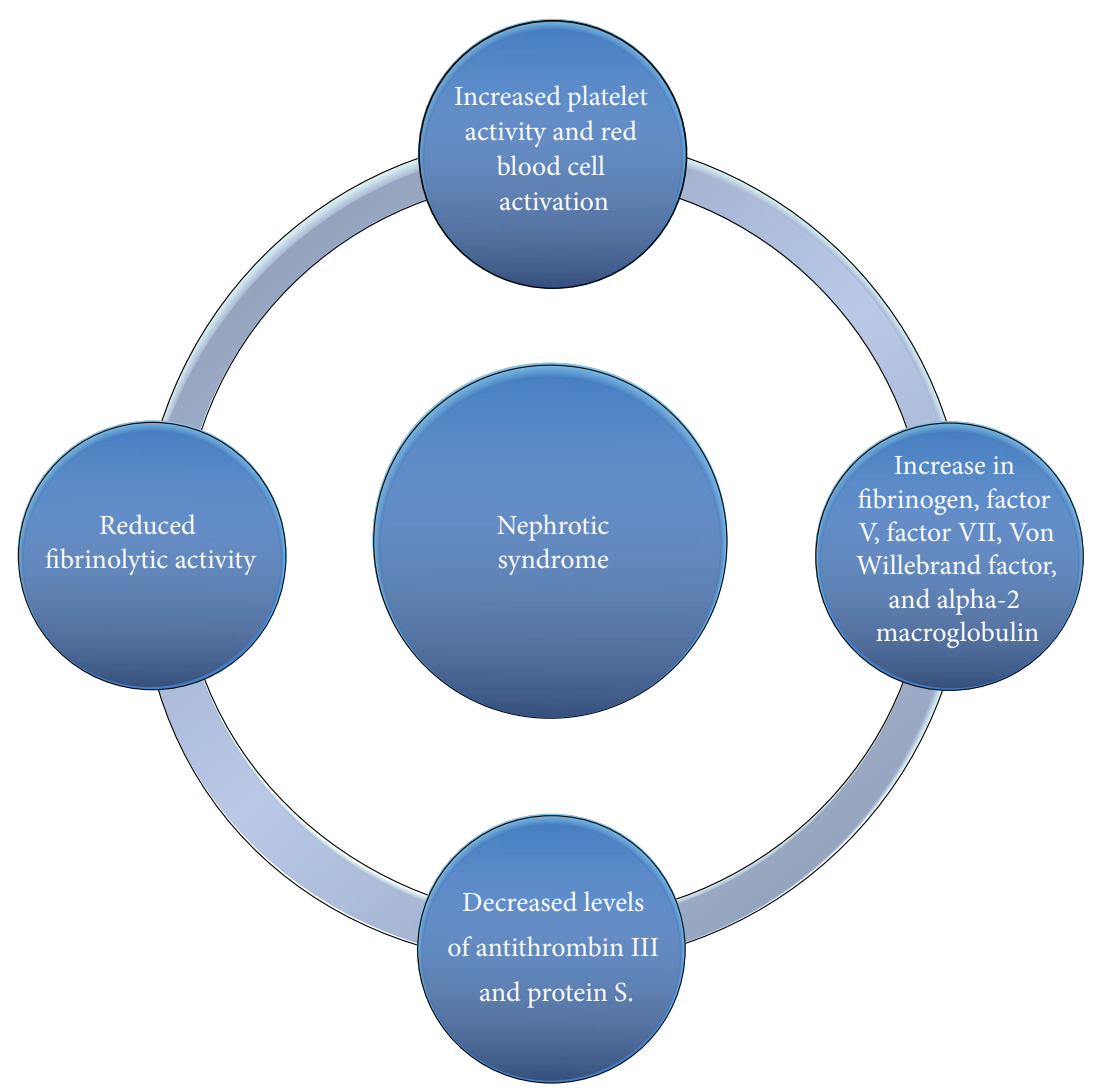

Figure 1: A simplified sketch on the pathogenesis of NS-related thrombotic risk.

TABLE 1: Wells score for DVT (adapted from [52]).

\begin{tabular}{ll}
\hline Variable & Points \\
\hline Active cancer (treatment ongoing or within the previous 6 months or palliative treatment) & 1 \\
Paralysis, paresis, or recent immobilization of the lower extremities & 1 \\
Recently bedridden for 3 days or more or major surgery within previous 12 weeks requiring general or regional anesthesia & 1 \\
Local tenderness along the distribution of the deep venous system & 1 \\
Entire leg swollen & 1 \\
Calf swelling $>3 \mathrm{~cm}$ compared to asymptomatic leg (measuring $10 \mathrm{~cm}$ below tibial tuberosity) & 1 \\
Pitting edema confined to the symptomatic leg & 1 \\
Nonvaricose collateral veins & 1 \\
Previously documented DVT & 1 \\
Alternative diagnosis at least as likely as DVT & -2 \\
\hline
\end{tabular}

\section{Scoring:}

$<0-$ low pretest probability.

$1-2-$ moderate pretest probability.

$\geq 3$ - high pretest probability.

[57]. Therefore, in some patients with NS and PE, the origin of pulmonary embolus is from the renal veins [57].

Another important issue is that a considerable number of patients with PE and NS are asymptomatic (at least 12\%), as demonstrated by Cherng et al. [49]. Thus, it is important to retain a clinical suspicion for VTE in patients with NS since even clinically silent and chronic PE may lead to a serious complication such as CTEPH [10].

\section{Prophylaxis against Increased Thrombotic Risk in Patients with NS}

It is essential to note that the scientific literature is scant, with no randomized data available on the topic of primary thrombotic prevention in patients with NS. Sarasin and Schifferli in their analysis demonstrated that patients with MN benefit from primary prophylactic anticoagulation [59]. Rostoker 
TABle 2: Wells score for PE (adapted from [54]).

\begin{tabular}{lc}
\hline Variable & Points \\
\hline Clinical signs and symptoms compatible with DVT & 3 \\
PE judged to be the most likely diagnosis & 3 \\
Surgery or bedridden for more than 3 days during the past 4 weeks & 1.5 \\
Previous DVT or PE & 1.5 \\
Heart rate > 100/minute & 1.5 \\
Hemoptysis & 1 \\
Active cancer (treatment ongoing or within the previous 6 months or palliative treatment) & 1 \\
\hline Scoring: & \\
$\leq 4-$ low pretest probability. & \\
4.5-6-moderate pretest probability. &
\end{tabular}

et al. studied 30 patients with NS to assess the utility of primary chemoprevention of thrombosis with low molecular weight heparin [60]. The study participants were followed for 13 months, and no thrombotic events or treatment side effects were observed. Nevertheless, given the small study sample, the lack of a control group, and the short follow-up period, it is impossible to translate their results into everyday clinical practice.

In a recent study, Lee et al. studied whether patients with $\mathrm{MN}$ benefit from primary prophylactic anticoagulation [61]. Patients were subdivided according to the levels of serum albumin into three groups: serum albumin $<3.0 \mathrm{mg} / \mathrm{dL}$, serum albumin $<2.5 \mathrm{mg} / \mathrm{dL}$, and serum albumin $<2.0 \mathrm{mg} / \mathrm{dL}$. As was discussed earlier in the text, patients with lower serum albumin (especially with serum albumin $<2.0 \mathrm{mg} / \mathrm{dL}$ ) represent a high risk group for thromboembolic events. Furthermore, these researchers stratified their bleeding risk using ATRIA score which was initially invented for patients with atrial fibrillation. It was demonstrated that patients who are at low risk of bleeding according to the ATRIA score would benefit from anticoagulation (benefit to risk ratio of $4.5: 1,5.2: 1$, and $13.1: 1$ for patients with serum albumin $<3.0 \mathrm{mg} / \mathrm{dL}$, serum albumin $<2.5 \mathrm{mg} / \mathrm{dL}$, and serum albumin $<2.0$, resp.). Patients who are at intermediate risk of bleeding according to the ATRIA score and serum albumin level $<2.0 \mathrm{mg} / \mathrm{dL}$ were found to have the benefit to risk ratio of $3.9: 1$ and patients with high bleeding risk should not be treated with prophylactic anticoagulation according to their results given high risk of serious bleeding (online calculator is available at http://www.unckidneycenter.org/gntools/gntools-team.html to calculate the benefit to risk ratio of anticoagulation according to their data). However, patients with hypoalbuminemia may be at increased risk of bleeding and, indeed, this parameter is a part of ATRIA score. However, this is unclear whether hypoalbuminemia is a true risk factor for bleeding in patients with NS (ATRIA score is primarily for patients with atrial fibrillation). The other limitations of this study are its retrospective analysis, no control group, and inclusion of patients only with MN. Therefore, the approach above cannot be recommended to patients with NS histology other than MN.
Medjeral-Thomas et al. retrospectively studied different thromboprophylactic regimens in 143 patients with NS (58 with MN, 45 with MCD, and 40 with FSGS) [62]. Patients were stratified according to their serum albumin levels (serum albumin $>3.0 \mathrm{mg} / \mathrm{dL}$, serum albumin $2-3 \mathrm{mg} / \mathrm{dL}$, and serum albumin $<2.0 \mathrm{mg} / \mathrm{dL}$ ). Patients with serum albumin $>3.0 \mathrm{mg} / \mathrm{dL}$ did not receive any prophylactic anticoagulation, patients with serum albumin $2.0-3.0 \mathrm{mg} / \mathrm{dL}$ received low dose aspirin $(75 \mathrm{mg} /$ day $)$, and patients with serum albumin $<2.0 \mathrm{mg} / \mathrm{dL}$ received either low dose of low molecular weight heparin or warfarin with a goal international normalized ratio of 1.5-2.5. Two patients developed venous thrombotic events within a week of starting anticoagulation, one patient on anticoagulation developed urgent gastrointestinal bleeding, and two patients on anticoagulation received elective blood transfusion for occult gastrointestinal bleeding. The major limitations of this study are its retrospective design and lack of the control group.

The question is, therefore, what can be done until solid data are available? First, it is essential to discuss the pros and cons of anticoagulation in all patients with NS (especially in patients with hypoalbuminemia), which should lead to informed patient decisions. Second, it is prudent to state that NS secondary to MN may impose a greater thrombotic risk. Third, other risk factors for DVT, VTE, and PE should be considered (e.g., a recent surgery or prior DVT/PE), and pharmacological anticoagulation should be commenced, unless being contraindicated. Fourth, it is important to consider compelling indications for pharmacological anticoagulation, such as atrial fibrillation. Fifth, statins, which are commonly used in patients with NS, may modestly decrease the risk of DVT [63]. Sixth, aspirin may be used to diminish the risk of VTE, as shown in recent studies published in the New England Journal of Medicine [64] and Clinical Journal of the American Society of Nephrology [62]. However, it is essential to mention that there are no data from studies of the utility of aspirin for thromboprophylaxis in patients with NS.

A much clearer situation is when the patient is found to have a thrombotic event, such as DVT, renal vein thrombosis, or PE. In such cases, active therapeutic pharmacological anticoagulation is warranted, unless being contraindicated [8]. In cases of PE with cardiovascular compromise and right 
ventricular dysfunction, which is also known as a massive $\mathrm{PE}$, there is a need for pharmacological thrombolysis or embolectomy if thrombolysis is contraindicated [56]. The data regarding elastic compression stockings and their impact on the incidence of VTE is lacking, but, given a low adverse effect profile, this approach may be recommended for some patients with NS who are at an increased risk of DVT and for patients with clear contraindications to anticoagulation [65]. It is important to note that the treatment approach is not different for patients with NS and the reader is referred to some well-written reviews on this topic $[6,66]$.

In patients with DVT who are not candidates for pharmacological anticoagulation, IVC filters should be placed [66]. Suprarenal IVC filters may be suitable for patients with NS given a greater incidence of renal vein thrombosis in this group [67]. It is important to keep in mind that IVC filters are associated with some complications, though being found in a small number of patients [68-70]. The list of potential complications of IVC filter insertion includes thrombotic complications such as thrombosis at the site of insertion, local complications such as hematoma formation at the insertion site, and filter associated complications such as filter migration, filter embolization, and erosion of the IVC.

Another question, which urgently needs prospective randomized data, is regarding the duration of anticoagulation (either for primary or secondary prevention and treatment) in patients with NS. A common belief is that patients with NS who are at remission for at least 2 years have decreased thrombotic risk. Indeed, as was discussed above, patients with normal or near normal serum albumin levels have minimal risk of thrombosis and do not benefit from pharmacological prophylactic anticoagulation. Another factor to consider is whether NS is secondary to cancer since the latter may further increase the risk of thrombosis [11].

We suggest that the overall risk assessment is essential and will provide some possible examples which clinicians may encounter in everyday clinical practice. The first theoretical case was a 35-year-old nonobese male with NS secondary to FSGS, without any additional risk factors for DVT and no history of a thrombotic event and albumin level of $2.1 \mathrm{~g} / \mathrm{L}$. When approaching such a case, it is important to keep in mind that FSGS is thought to be less associated with DVT/VTE than NS secondary to MN. An albumin level $\leq 2.0 \mathrm{~g} / \mathrm{L}$ has been found to impose increased thrombotic risk in such patients (discussed in previous sections). Therefore, in such cases, it is essential to educate the patient on the condition and its potential thrombotic risk, benefits versus risks of anticoagulation, and any ancillary therapies which might modestly reduce thrombotic risk (such as statins and low dose aspirin). Furthermore, it should be clearly conveyed to the patient that there is no randomized prospective data regarding anticoagulation in patients with NS and FSGS in particular. Thus, a patient's informed decision is the key in such a scenario; however, from the current evidence standpoint, we would not start this patient on pharmacological anticoagulation but may offer low dose aspirin for thromboprophylaxis if he has no contraindications to antiplatelet therapy.
The second theoretical case was a 47-year-old female with NS secondary to $\mathrm{MN}$, an albumin level of $1.7 \mathrm{~g} / \mathrm{l}$, congestive heart failure, and atrial fibrillation. This case is relatively straightforward since the patient has many compelling indications for pharmacological anticoagulation, such as atrial fibrillation and an albumin level of 1.7 in a setting of MN. Furthermore, the presence of atrial fibrillation obviates the need to consider serum albumin in this patient. Therefore, such patients should be counseled on their substantially increased risk of a thrombotic event (either venous or embolic stroke). The third theoretical case was a 50-year-old female with NS secondary to MCD, with no compelling indications for thromboprophylaxis, risk factors for DVT, or prior history of DVT and an albumin level of $1.7 \mathrm{~g} / \mathrm{L}$. In this case, the patient should be counseled that based on the albumin level she is probably at increased risk for DVT. However, randomized scientific data are lacking and the decision of whether to anticoagulate or not is the most challenging in this particular case. One may consider extrapolating the approach used by Lee et al. [61] and estimate the bleeding risk using ATRIA score. However, it is essential to remember that study by Lee did not include patients with MCD. Therefore, detailed patient education and mutual decision-making are especially important in this case.

Finally, it is essential to address the question regarding the duration of anticoagulation. Again, there are no data on how long anticoagulation should be continued in patients with NS. One potential approach is to continue anticoagulation (if no contraindication) till serum albumin levels normalize and the patients with NS achieve remission. Nevertheless, we believe that overall and thorough risk factor assessment is essential. For example, we will use indefinite anticoagulation (unless contraindicated) in cases of NS with low albumin levels and a compelling indication for anticoagulation such as atrial fibrillation or multiple VTE.

\section{Conclusion}

Patients with NS are at an increased risk of thrombotic events; DVT, RVT, and PE are quite common in patients with NS. It is important to note that NS secondary to MN may impose a greater thrombotic risk for unclear reasons. Increased platelet activation, enhanced RBC aggregation, and an imbalance between procoagulant and anticoagulant factors are thought to underlie the excessive thrombotic risk in patients with NS. The current scientific literature does not provide a solid answer on the role of primary thromboprophylaxis among patients with NS. One possible exception is patients with $\mathrm{MN}$ and hypoalbuminemia who are at low risk for bleeding. A thorough approach which includes accounting for all additional thrombotic risk factors is, therefore, essential. Patient counseling regarding the pros and cons of anticoagulation is of paramount importance. Future studies should address the question regarding the utility of primary thromboprophylaxis. 


\section{Conflict of Interests}

The authors declare that there is no conflict of interests regarding the publication of this paper.

\section{References}

[1] J. S. Cameron and J. Hicks, "The origins and development of the concept of a 'nephrotic syndrome," American Journal of Nephrology, vol. 22, no. 2-3, pp. 240-247, 2002.

[2] R. P. Hull and D. J. A. Goldsmith, "Nephrotic syndrome in adults," British Medical Journal, vol. 336, no. 7654, pp. 1185-1189, 2008.

[3] R. J. Glassock, "Attending rounds: an older patient with nephrotic syndrome," Clinical Journal of the American Society of Nephrology, vol. 7, no. 4, pp. 665-670, 2012.

[4] A. Meyrier, "Focal and segmental glomerulosclerosis: multiple pathways are involved," Seminars in Nephrology, vol. 31, no. 4, pp. 326-332, 2011.

[5] J. J. Weening, V. D. D’Agati, M. M. Schwartz et al., "The classification of glomerulonephritis in systemic lupus erythematosus revisited," Journal of the American Society of Nephrology, vol. 15, no. 3, pp. 241-250, 2004.

[6] S. Takach Lapner and C. Kearon, "Diagnosis and management of pulmonary embolism," British Medical Journal, vol. 346, p. f757, 2013.

[7] T. Pulido, A. Aranda, M. A. Zevallos et al., "Pulmonary embolism as a cause of death in patients with heart disease: an autopsy study," Chest, vol. 129, no. 5, pp. 1282-1287, 2006.

[8] J. A. Heit, W. Michael O’Fallon, T. M. Petterson et al., "Relative impact of risk factors for deep vein thrombosis and pulmonary embolism: a population-based study," Archives of Internal Medicine, vol. 162, no. 11, pp. 1245-1248, 2002.

[9] B. Zöller, X. Li, J. Sundquist, and K. Sundquist, "Risk of pulmonary embolism in patients with autoimmune disorders: a nationwide follow-up study from Sweden," The Lancet, vol. 379, no. 9812, pp. 244-249, 2012.

[10] A. E. Mirrakhimov and N. S. Hill, "Primary antiphospholipid syndrome and pulmonary hypertension," Current Pharmaceutical Design, vol. 20, pp. 545-551, 2014.

[11] M. N. Lauw, F. F. van Doormaal, S. Middeldorp, and H. R. Buller, "Cancer and venous thrombosis: current comprehensions and future perspectives," Seminars in Thrombosis and Hemostasis, vol. 39, pp. 507-514, 2013.

[12] G. Remuzzi, G. Mecca, D. Marchesi et al., "Platelet hyperaggregability and the nephrotic syndrome," Thrombosis Research, vol. 16, no. 3-4, pp. 345-354, 1979.

[13] E. Walter, D. Deppermann, K. Andrassy, and J. Koderisch, "Platelet hyperaggregability as a consequence of the nephrotic syndrome," Thrombosis Research, vol. 23, no. 6, pp. 473-479, 1981.

[14] V. Sirolli, E. Ballone, D. Garofalo et al., "Platelet activation markers in patients with nephrotic syndrome: a comparative study of different platelet function tests," Nephron, vol. 91, no. 3, pp. 424-430, 2002.

[15] A. Mittal, K. C. Aggarwal, S. Saluja, A. Aggarwal, and B. Sureka, "Platelet functions and coagulation changes in Indian children with nephrotic syndrome," Journal of Clinical and Diagnostic Research, vol. 7, pp. 1647-1650, 2013.

[16] H. J. Ting, J. P. Murad, E. V. Espinosa, and F. T. Khasawneh, "Thromboxane A2 receptor: biology and function of a peculiar receptor that remains resistant for therapeutic targeting," Journal of Cardiovascular Pharmacology and Therapeutics, vol. 17, pp. 248-259, 2012.

[17] A. M. Astudillo, D. Balgoma, M. A. Balboa, and J. Balsinde, "Dynamics of arachidonic acid mobilization by inflammatory cells," Biochimica et Biophysica Acta, vol. 1821, no. 2, pp. 249256, 2012.

[18] N. Yoshida and N. Aoki, "Release of arachidonic acid from human platelets. A key role for the potentiation of platelet aggregability in normal subjects as well as in those with nephrotic syndrome," Blood, vol. 52, no. 5, pp. 969-977, 1978.

[19] C. A. Jackson, M. Greaves, and A. D. Patterson, "Relationship between platelet aggregation, thromboxane synthesis and albumin concentration in nephrotic syndrome," British Journal of Haematology, vol. 52, no. 1, pp. 69-77, 1982.

[20] A. Schieppati, P. Dodesini, and A. Benigni, "The metabolism of arachidonic acid by platelets in nephrotic syndrome," Kidney International, vol. 25, no. 4, pp. 671-676, 1984.

[21] D. P. Mikhailidis and E. S. Ganotakis, "Plasma albumin and platelet function: relevance to atherogenesis and thrombosis," Platelets, vol. 7, no. 3, pp. 125-137, 1996.

[22] J. J. Zwaginga, H. A. Koomans, J. J. Sixma, and T. J. Rabelink, "Thrombus formation and platelet-vessel wall interaction in the nephrotic syndrome under flow conditions," Journal of Clinical Investigation, vol. 93, no. 1, pp. 204-211, 1994.

[23] S. J. Shattil, R. Anaya-Galindo, and J. Bennett, "Platelet hypersensitivity induced by cholesterol incorporation," Journal of Clinical Investigation, vol. 55, no. 3, pp. 636-643, 1975.

[24] C. Machleidt, T. Mettang, E. Stärz, J. Weber, T. Risler, and U. Kuhlmann, "Multifactorial genesis of enhanced platelet aggregability in patients with nephrotic syndrome," Kidney International, vol. 36, no. 6, pp. 1119-1124, 1989.

[25] P. Ozanne, R. B. Francis, and H. J. Meiselman, "Red blood cell aggregation in nephrotic syndrome," Kidney International, vol. 23, no. 3, pp. 519-525, 1983.

[26] T. Bohler, O. Linderkamp, A. Leo, A.-M. Wingen, and K. Scharer, "Increased aggregation with normal surface charge and deformability of red blood cells in children with nephrotic syndrome," Clinical Nephrology, vol. 38, no. 3, pp. 119-124, 1992.

[27] M. M. Patnaik and S. Moll, "Inherited antithrombin deficiency: a review," Haemophilia, vol. 14, no. 6, pp. 1229-1239, 2008.

[28] R. H. Kauffmann, J. J. Veltkamp, N. H. van Tilburg, and L. A. van Es, "Acquired antithrombin III deficiency and thrombosis in the nephrotic syndrome," American Journal of Medicine, vol. 65, no. 4, pp. 607-613, 1978.

[29] K. A. Jørgensen and E. Stoffersen, "Antithrombin III and the nephrotic syndrome," Scandinavian Journal of Haematology, vol. 22, no. 5, pp. 442-448, 1979.

[30] N. D. Vaziri, P. Paule, and J. Toohey, "Acquired deficiency and urinary excretion of antithrombin III in nephrotic syndrome," Archives of Internal Medicine, vol. 144, no. 9, pp. 1802-1803, 1984.

[31] T.-Y. Chen, C.-C. Huang, and C.-J. Tsao, "Hemostatic molecular markers in nephrotic syndrome," American Journal of Hematology, vol. 44, no. 4, pp. 276-279, 1993.

[32] Z. Bereczky, K. B. Ková, and L. Muszbek, "Protein C and protein $S$ deficiencies: similarities and differences between two brothers playing in the same game," Clinical Chemistry and Laboratory Medicine, vol. 48, no. 1, pp. S53-S66, 2010.

[33] F. G. Cosio, C. Harker, M. A. Batard, J. T. Brandt, and J. H. Griffin, "Plasma concentrations of the natural anticoagulants protein $\mathrm{C}$ and protein $\mathrm{S}$ in patients with proteinuria," The 
Journal of Laboratory and Clinical Medicine, vol. 106, no. 2, pp. 218-222, 1985.

[34] S. Vigano-D’Angelo, A. D’Angelo, C. E. Kaufman Jr., C. Sholer, C. T. Esmon, and P. C. Comp, "Protein S deficiency occurs in the nephrotic syndrome," Annals of Internal Medicine, vol. 107, no. 1, pp. 42-47, 1987.

[35] P. C. Comp, D. Doray, D. Patton, and C. T. Esmon, "An abnormal plasma distribution of protein $\mathrm{S}$ occurs in functional protein $\mathrm{S}$ deficiency," Blood, vol. 67, no. 2, pp. 504-508, 1986.

[36] N. Öskayin, S. Mir, and K. Kavakli, "Hypercoagulability risk factors in children with minimal change disease and the protective role of protein-C activity," International Urology and Nephrology, vol. 36, no. 4, pp. 599-603, 2004.

[37] M. M. Al-Mugeiren, A. G. M. Abdel Gader, S. A. Al-Rasheed, and A. A. Al-Salloum, "Tissue factor pathway inhibitor in childhood nephrotic syndrome," Pediatric Nephrology, vol. 21, no. 6, pp. 771-777, 2006.

[38] M. G. M. de Sain-van der Velden, G. A. Kaysen, K. de Meer et al., "Proportionate increase of fibrinogen and albumin synthesis in nephrotic patients: measurements with stable isotopes," Kidney International, vol. 53, no. 1, pp. 181-188, 1998.

[39] J. Loscalzo, "Venous thrombosis in the nephrotic syndrome," The New England Journal of Medicine, vol. 368, pp. 956-958, 2013.

[40] N. Schlegel, "Thromboembolic risks and complications in nephrotic children," Seminars in Thrombosis and Hemostasis, vol. 23, no. 3, pp. 271-280, 1997.

[41] J. C. M. Meijers, W. L. H. Tekelenburg, B. N. Bouma, R. M. Bertina, and F. R. Rosendaal, "High levels of coagulation factor $\mathrm{XI}$ as a risk factor for venous thrombosis," The New England Journal of Medicine, vol. 342, no. 10, pp. 696-701, 2000.

[42] C. Thomson, C. D. Forbes, C. R. M. Prentice, and A. C. Kennedy, "Changes in blood coagulation and fibrinolysis in the nephrotic syndrome," Quarterly Journal of Medicine, vol. 43, no. 171, pp. 399-407, 1974.

[43] P. Svenningsen, C. Bistrup, U. G. Friis et al., "Plasmin in nephrotic urine activates the epithelial sodium channel," Journal of the American Society of Nephrology, vol. 20, no. 2, pp. 299-310, 2009.

[44] T. Soulat, S. Loyau, V. Baudouin et al., "Effect of individual plasma lipoprotein(a) variations in vivo on its competition with plasminogen for fibrin and cell binding: an in vitro study using plasma from children with idiopathic nephrotic syndrome," Arteriosclerosis, Thrombosis, and Vascular Biology, vol. 20, no. 2, pp. 575-584, 2000.

[45] Y. Yoshida, H. Shiiki, M. Iwano et al., "Enhanced expression of plasminogen activator inhibitor 1 in patients with nephrotic syndrome," Nephron, vol. 88, no. 1, pp. 24-29, 2001.

[46] J.-P. Collet, Z. Mishal, C. Lesty et al., "Abnormal fibrin clot architecture in nephrotic patients is related to hypofibrinolysis: Influence of plasma biochemical modifications. A possible mechanism for the high thrombotic tendency?" Thrombosis and Haemostasis, vol. 82, no. 5, pp. 1482-1489, 1999.

[47] S. J. Barbour, A. Greenwald, O. Djurdjev et al., "Disease-specific risk of venous thromboembolic events is increased in idiopathic glomerulonephritis," Kidney International, vol. 81, no. 2, pp. 190-195, 2012.

[48] S. Lionaki, V. K. Derebail, S. L. Hogan et al., "Venous thromboembolism in patients with membranous nephropathy," Clinical Journal of the American Society of Nephrology, vol. 7, no. 1, pp. 43-51, 2012.
[49] S.-C. Cherng, W.-S. Huang, Y.-F. Wang, S.-P. Yang, and Y.-F. Lin, "The role of lung scintigraphy in the diagnosis of nephrotic syndrome with pulmonary embolism," Clinical Nuclear Medicine, vol. 25, no. 3, pp. 167-172, 2000.

[50] U. Kuhlmann, J. Steurer, A. Bollinger, G. Pouliadis, J. Briner, and W. Siegenthaler, "Incidence and clinical significance of thromboses and thrombo-embolic complications in nephrotic syndrome patients," Schweizerische Medizinische Wochenschrift, vol. 111, pp. 1034-1040, 1981.

[51] A. A. Eddy and J. M. Symons, "Nephrotic syndrome in childhood," The Lancet, vol. 362, no. 9384, pp. 629-639, 2003.

[52] J. Wilbur and B. Shian, "Diagnosis of deep venous thrombosis and pulmonary embolism," American Family Physician, vol. 86, pp. 913-919, 2012.

[53] P. S. Wells, D. R. Anderson, M. Rodger et al., "Evaluation of Ddimer in the diagnosis of suspected deep-vein thrombosis," The New England Journal of Medicine, vol. 349, no. 13, pp. 1227-1235, 2003.

[54] R. Oudega, A. W. Hoes, and K. G. M. Moons, "The Wells rule does not adequately rule out deep venous thrombosis in primary care patients," Annals of Internal Medicine, vol. 143, no. 2, pp. 100-107, 2005.

[55] A. van Belle, H. R. Büller, M. V. Huisman et al., "Effectiveness of managing suspected pulmonary embolism using an algorithm combining clinical probability, D-dimer testing, and computed tomography," Journal of the American Medical Association, vol. 295, no. 2, pp. 172-179, 2006.

[56] N. Kucher and S. Z. Goldhaber, "Management of massive pulmonary embolism," Circulation, vol. 112, no. 2, pp. e28-e32, 2005.

[57] F. Kayali, R. Najjar, F. Aswad, F. Matta, and P. D. Stein, "Venous thromboembolism in patients hospitalized with nephrotic syndrome," American Journal of Medicine, vol. 121, no. 3, pp. 226230, 2008.

[58] D. Suri, J. Ahluwalia, A. K. Saxena et al., "Thromboembolic complications in childhood nephrotic syndrome: a clinical profile," Clinical and Experimental Nephrology, 2013.

[59] F. P. Sarasin and J. A. Schifferli, "Prophylactic oral anticoagulation in nephrotic patients with idiopathic membranous nephropathy," Kidney International, vol. 45, no. 2, pp. 578-585, 1994.

[60] G. Rostoker, I. Durand-Zaleski, M. Petit-Phar et al., "Prevention of thrombotic complications of the nephrotic syndrome by the low-molecular weight heparin Enoxaparin," Nephron, vol. 69, no. 1, pp. 20-28, 1995.

[61] T. Lee, A. K. Biddle, S. Lionaki et al., "Personalized prophylactic anticoagulation decision analysis in patients with membranous nephropathy," Kidney International, 2013.

[62] N. Medjeral-Thomas, S. Ziaj, M. Condon et al., "Retrospective analysis of a novel regimen for the prevention of venous thromboembolism in nephrotic syndrome," Clinical Journal of the American Society of Nephrology, vol. 9, no. 3, pp. 478-483, 2014.

[63] L. Li, T. Sun, P. Zhang, J. Tian, and K. Yang, "Statins for primary prevention of venous thromboembolism," Cochrane Database of Systematic Reviews, vol. 12, p. CD008203, 2011.

[64] C. Becattini, G. Agnelli, A. Schenone et al., "Aspirin for preventing the recurrence of venous thromboembolism," The New England Journal of Medicine, vol. 366, pp. 1959-1967, 2012.

[65] A. Sachdeva, M. Dalton, S. V. Amaragiri, and T. Lees, "Elastic compression stockings for prevention of deep vein thrombosis," 
Cochrane Database of Systematic Reviews, vol. 7, p. CD001484, 2010.

[66] O. Osinbowale, L. Ali, and Y.-W. Chi, "Venous thromboembolism: a clinical review," Postgraduate Medicine, vol. 122, no. 2, pp. 54-65, 2010.

[67] L. J. Greenfield, K. J. Cho, M. C. Proctor, M. Sobel, S. Shah, and J. Wingo, "Late results of suprarenal Greenfield vena cava filter placement," Archives of Surgery, vol. 127, no. 8, pp. 969-973, 1992.

[68] M. Nazzal, E. Chan, M. Nazzal et al., "Complications related to inferior vena cava filters: a single-center experience," Annals of Vascular Surgery, vol. 24, no. 4, pp. 480-486, 2010.

[69] L. F. Angel, V. Tapson, R. E. Galgon, M. I. Restrepo, and J. Kaufman, "Systematic review of the use of retrievable inferior vena cava filters," Journal of Vascular and Interventional Radiology, vol. 22, no. 11, pp. 1522-1530, 2011.

[70] S. Sarosiek, M. Crowther, and J. M. Sloan, "Indications, complications, and management of inferior vena cava filters: the experience in 952 patients at an academic hospital with a level I trauma center," JAMA Internal Medicine, vol. 173, pp. 513-517, 2013. 


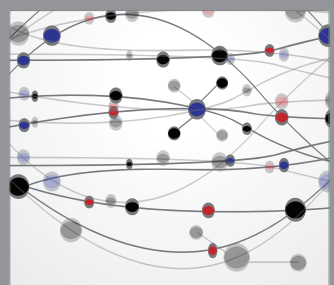

The Scientific World Journal
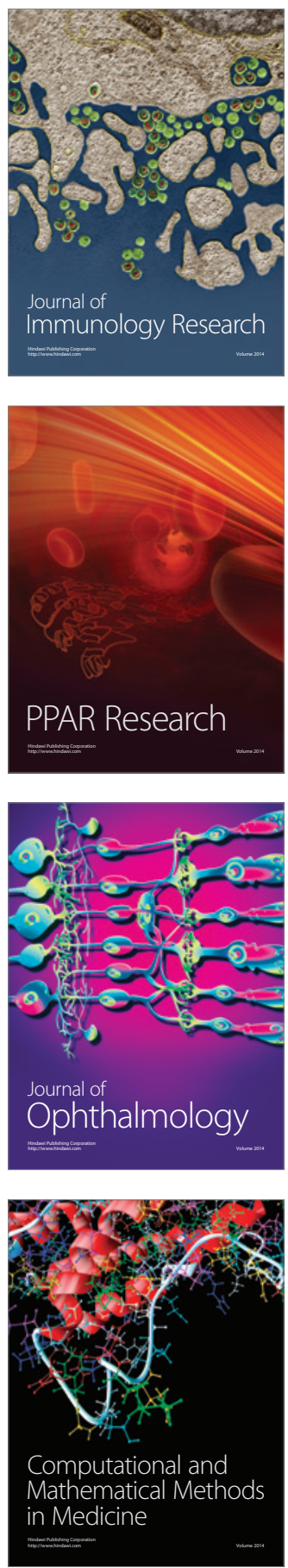

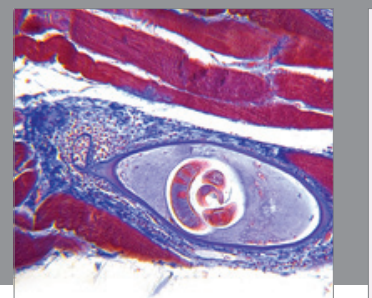

Gastroenterology

Research and Practice
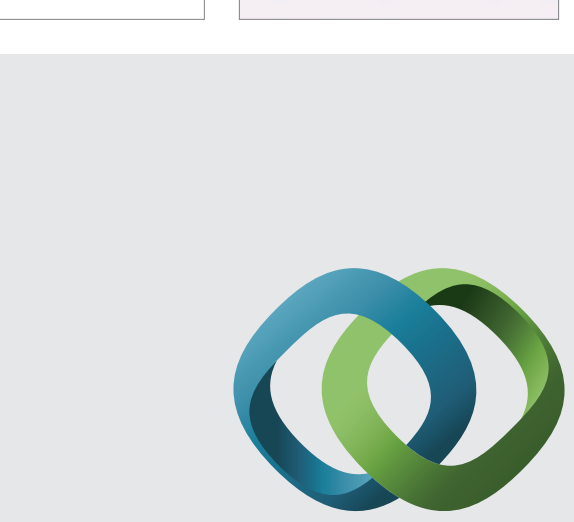

\section{Hindawi}

Submit your manuscripts at

http://www.hindawi.com
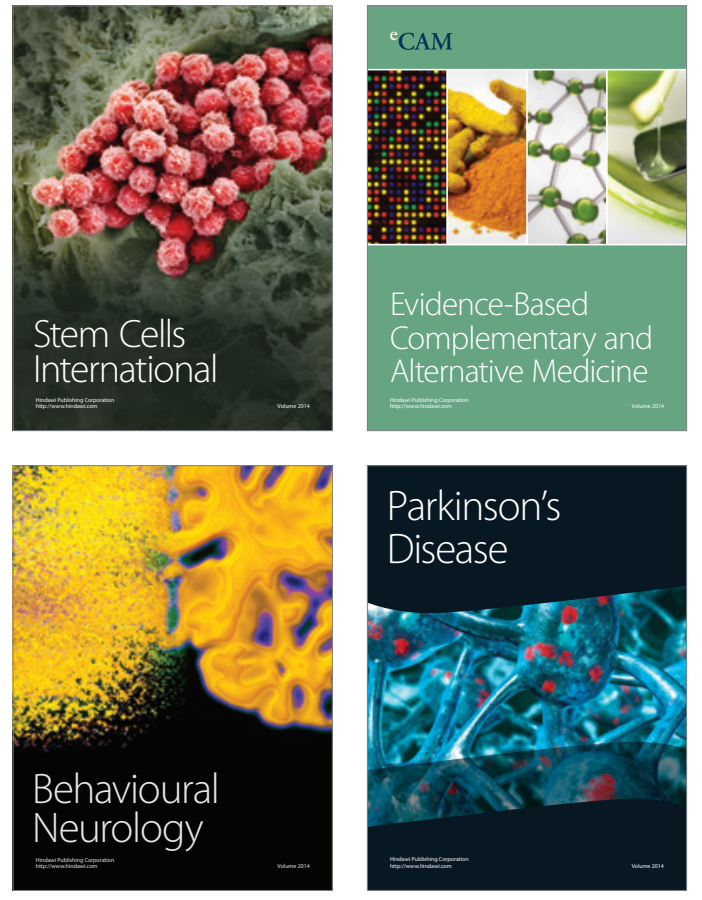
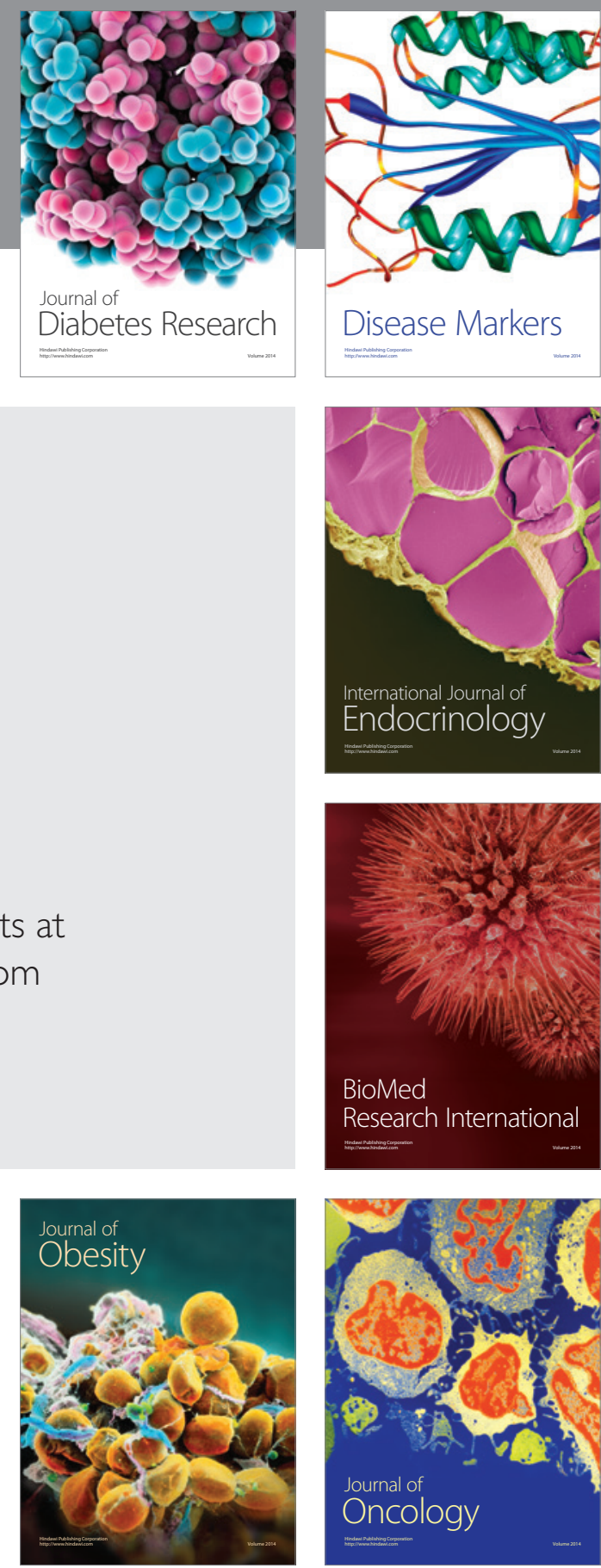

Disease Markers
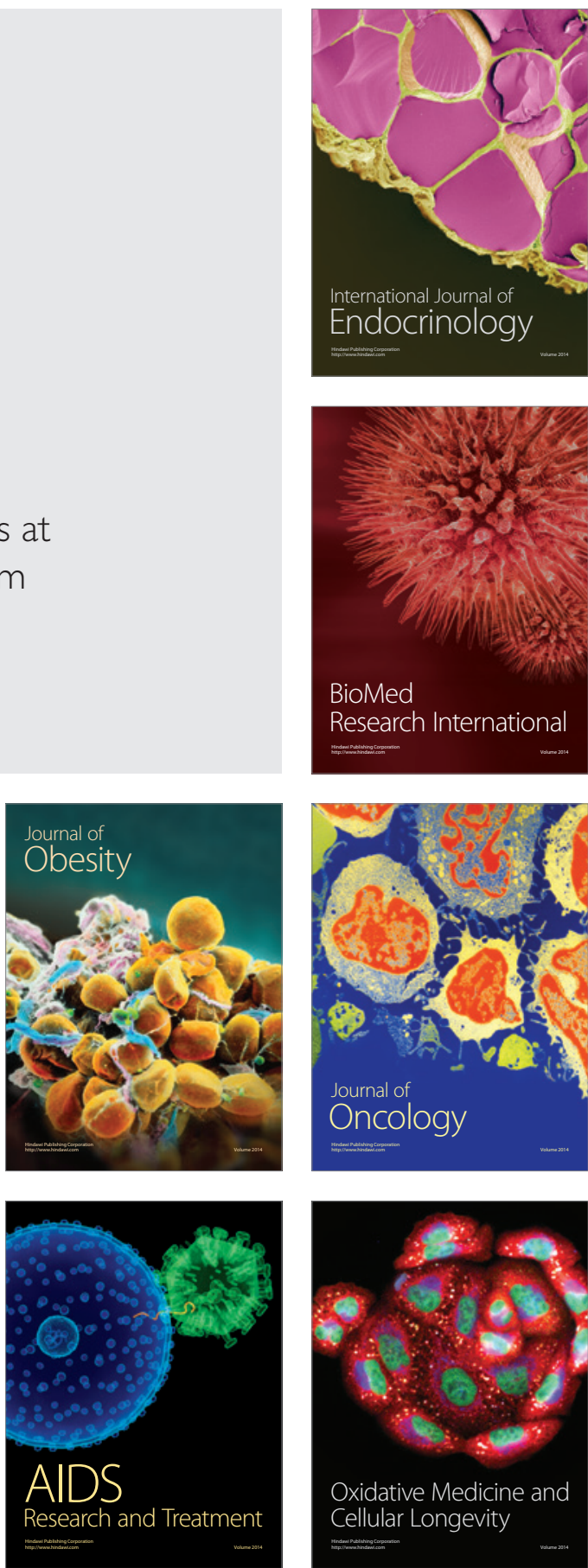